\title{
ERG expression in prostate cancer biopsies with and without high-grade prostatic intraepithelial neoplasia: a study in Jordanian Arab patients
}

This article was published in the following Dove Press journal: Research and Reports in Urology

Najla Aldaoud (1)
Ashley Graboski-Bauer
Nour Abdo
Samir Al Bashir ${ }^{1,2}$
Ashraf O Oweis
Hanadi Ebwaini
Yousef Hasen ${ }^{1,2,6}$
Rami Alazab
Kiril Trpkov

'Department of Pathology and
Microbiology, King Abdullah University
Hospital, Irbid, Jordan; ${ }^{2}$ Jordan University
of Science and Technology, Irbid, Jordan;
${ }^{3}$ Department of Teacher Education,
University of Texas, El Paso, TX, USA;
${ }^{4}$ Department of Public Health, Jordan
University of Science and Technology,
Irbid, Jordan; ${ }^{5}$ Division of Nephrology,
Department of Medicine, Jordan
University of Science and Technology,
Irbid, Jordan; ${ }^{6}$ Attasami Diagnostic
Center, Tripoli, Libya; ${ }^{7}$ Division of
Urology, Jordan University of Science and
Technology, Irbid, Irbid; ${ }^{8}$ Department of
Pathology and Laboratory Medicine,
University of Calgary, Calgary, AB,
Canada

Correspondence: Najla Aldaoud Department of Pathology and Microbiology, Jordan University of Science and Technology and King Abdullah University Hospital, PO Box 3030, Irbid 22110, Jordan

Tel +96227200600 ext 41328

Fax +9622 7200626

Email naglaadaoud@yahoo.com
Background: High-grade prostatic intraepithelial neoplasia (HGPIN) is the most likely precancerous lesion for prostatic adenocarcinoma (PCa). Recent molecular studies have shown that HGPIN can harbor TMPRSS2-ERG fusion, a genetic marker also associated with $\mathrm{PCa}$, which may provide an additional risk stratification tool for HGPIN, especially when present as an isolated lesion. Our aim was to assess the frequency of HGPIN and ERG expression in a cohort of prostatic needle core biopsies from Jordanian-Arab patients with $\mathrm{PCa}$.

Materials and methods: We studied 109 needle core biopsies from patients with PCa. Clinical data, including age and preoperative prostate specific antigen (PSA) level, were obtained from patients' medical records.

Results: HGPIN was present in 31 (28.4\%) of the 109 cases. Of the HGPIN cases, 13 (41.9\%) expressed ERG immunostain. ERG expression in HGPIN was independent of patient age at presentation $(P=0.4)$, pre-operative PSA $(P=0.9)$, and the grade, using the novel Grade Groups $(P=0.5)$.

Conclusion: The frequency of HGPIN in our cohort appears similar to the one found in the Western patient populations and demonstrates a comparable frequency of ERG expression in these lesions.

Keywords: high-grade prostatic intraepithelial neoplasia, prostate carcinoma, ERG, immunohistochemistry

\section{Introduction}

High-grade prostatic intraepithelial neoplasia (HGPIN) is considered the most accepted precursor lesion to prostatic adenocarcinoma (PCa). ${ }^{1,2}$ Many studies have attempted to identify clinical, pathological, or molecular factors that can increase the predictive value of HGPIN and stratify patients at higher risk of developing subsequent PCa. Some of the published studies failed to find significant utility of these factors in predicting $\mathrm{PCa}^{3}$ On the other hand, other studies found that presence of HGPIN, patient age, and serum PSA were significant predictors of cancer, particularly when taken together. ${ }^{4}$ Moreover, multifocality of HGPIN and the number of cores involved were found to predict a higher risk of subsequent cancer on follow-up. ${ }^{5,6}$

Several genetic biomarkers have been proposed that may aid in the diagnosis and clinical management of PCa. ${ }^{7}$ TMPRSS2-ERG fusion is the most common genetic alteration found in $\mathrm{PCa}$, with frequency of $50 \%-70 \% .^{7-9}$ The frequency of ERG expression in $\mathrm{PCa}$ in the Jordanian-Arab population was found to be lower 
than that documented in North America. We previously studied 193 patieints with $\mathrm{PCa}$, and 64 (33.2\%) cases showed ERG expression. ${ }^{10}$ HGPIN adjacent to or intermingled with PCa was also found to harbor TMPRSS2ERG fusion in $0-50 \% .{ }^{11-20}$ Some studies have shown higher expression of TMPRSS2-ERG fusion in PCa to be associated with more aggressive disease, and thus suggested that testing for this genetic alterationmay be of prognostic utility. ${ }^{10,21-23}$ Other studies, however, have shown no prognostic value for ERG expression in PCa. ${ }^{13,18,24}$ With regard to isolated ERG-positive HGPIN, it has been found that subsequent PCa detected in these patients was mostly of Gleason Score 6, but there was no significant association between ERG expression in HGPIN with either the age of the patient at presentation or Gleason Score. ${ }^{14,25}$

To the best of our knowledge, no studies have investigated the frequency of HGPIN in Arab or Jordanian patients with PCa nor the frequency of ERG expression in these lesions. Therefore, the aims of our study were to investigate the frequency of HGPIN in a cohort of patients with PCa from our region and to assess the frequency of ERG expression in HGPIN lesions in this cohort.

\section{Methods}

\section{Patients and tissue samples}

We retrieved 109 consecutive prostatic needle-core biopsies with a diagnosis of PCa from the archives of King Abdullah University Hospital, obtained during 2005-2014. All cases were independently reviewed by two urologic pathologists (NA and SA) for the presence of HGPIN. Accompanying invasive cancers were classified according to the guidelines of the 2005 and 2014 International Society of Urological Pathology (ISUP) consensus conferences. $^{26,27}$ Of note, this does not represent a typical prostate specific antigen (PSA)-screened cohort, as often reported in the West, because we retrieved only two cases of HGPIN without PCa (not included in the study), and all other cases demonstrated both HGPIN and PCa. This reflects potentially a selection bias in this population, in which biopsies are performed in patients with more advanced disease. One representative section was selected from each case, containing PCa together with HGPIN. Clinical data, including age and preoperative PSA level, were obtained from patients' medical records. Of note, in our practice prostate biopsies are routinely submitted in two containers (right and left side), with five to six cores from one side submitted in the same container.

\section{Immunohistochemistry for ERG}

Immunohistochemistry (IHC) was performed on $4-\mu \mathrm{m}$ sections using a Dako autostainer-Plus (Dako, Denmark) and following a standard protocol. A monoclonal mouse antihuman ERG antibody (clone 9FY; Biocare Medical, Concord, CA, USA) at a dilution of 1:100 was used in this study. After the tissue had been dewaxed, antigen retrieval was carried out in a PT-Link (Dako, Denmark) using a high-pH buffer for 20 minutes. The slides were first washed with PBS and then blocked by $2.5 \%$ hydrogen peroxide. Then, sections were incubated with ERG (CM421C) for 30 minutes at room temperature. Signal detection was carried out using Flex dual link-detection kit (secondary antibody and DAB system K;8000; Dako, Denmark).

IHC staining for ERG was assessed in comparison to stromal endothelial cell reactivity, which was considered a positive internal control. Adjacent benign prostatic glands served as an internal negative control. A result was considered positive if any lesional cells showed nuclear positivity and negative if no lesional cells were reactive (Figure 1).

\section{Statistical analysis}

All analyses for dependent and independent variables were performed using SAS version 9.2; SAS institute, Cary, NC, U.S.A.). Frequencies and percentages were used for categorical variables (such as Gleason scores) and summary descriptive measures (means, ranges, and medians) were used for continuous variables (such as age). Statistical associations between expression of ERG (as a categorical variable) and continuous clinicopathological parameters (age and PSA at the time of diagnosis) were tested using Student's $t$-test, and with categorical parameters (Gleason sum) using Pearson's $\chi^{2}$. A $P<0.05$ was considered significant.

\section{Results}

\section{Patient characteristics}

Clinical and pathological characteristics of the entire cohort are summarized in Table 1. The mean number of sampled cores per biopsy was ten (range three to 20). The mean tumor volume was $46 \%$ (range $2 \%-95 \%$ ). The mean age of patients included in this cohort was 77.4 years (range 49-99 years). 


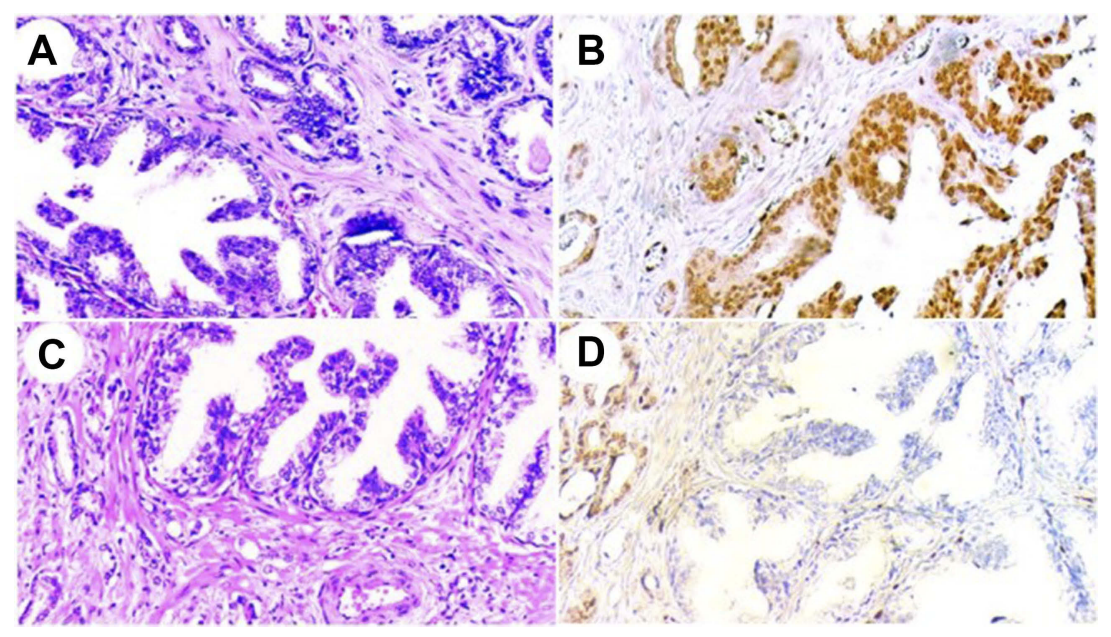

Figure I H\&E and corresponding ERG immunostaining in high-grade prostatic intraepithelial neoplasia (HGPIN) with adjacent invasive adenocarcinoma.

Notes: (A) HGPIN lesion at the lower left with accompanying invasive adenocarcinoma at the upper right (H\&E, 200x). (B) ERG immunostain for the same case, showing positive staining in both HGPIN (lower right) and the invasive component (upper left)(200x). (C) Another case with HGPIN (upper right) and adjacent small malignant glands (lower left) (H\&E, 200x). (D) ERG immunostain for the same case, showing negative staining in HGPIN (lower right), while the accompanying invasive component was positive (upper left) (200x).

Table I Clinicopathological characteristics and biopsy scores of Jordanian Arab prostate cancer patients

\begin{tabular}{|l|l|}
\hline Clinicopathologic variable & Value \\
\hline $\begin{array}{l}\text { Age at presentation } \\
\text { mean (range) }\end{array}$ & $77.4(49-99)$ \\
median (IQR) & $74.0(69.5-81.5)$ \\
\hline $\begin{array}{l}\text { Baseline PSA ng/ml } \\
\text { mean (range) }\end{array}$ & $276.2(1.5-6758)$ \\
median (IQR) & $32.1(15-150)$ \\
\hline$\leq$ I0 ng/ml, n (\%) & $15(13.8 \%)$ \\
mean (range) & $5.6(1.5-9.4)$ \\
median (IQR) & $5.8(5.2-6.6)$ \\
\hline$>10$ to 20 ng/ml, n (\%) & $22(20.2 \%)$ \\
mean (range) & $15.8(10.8-20)$ \\
median (IQR) & $15.5(13.4-18.2)$ \\
\hline$>20$ ng/ml, n (\%) & $59(54.1 \%)$ \\
mean (range) & $442.2(20.8-6758)$ \\
median (IQR) & $138.1(38.4-358.1)$ \\
\hline Unknown, n (\%) & $13(11.9 \%)$ \\
\hline Gleason Score (ISUP 20I4) & $16(14.7 \%)$ \\
$3+3$ (Grade Group I) & $14(12.8 \%)$ \\
$3+4$ (Grade Group 2) & $11(10.1 \%)$ \\
$4+3$ (Grade Group 3) & $34(31.2 \%)$ \\
$4+4$ (Grade Group 4) & $34(31.2 \%)$ \\
$4+5 / 5+5$ (Grade Group 5) & \\
\hline
\end{tabular}

Abbreviation: ISUP, International Society of Urological Pathology.

Preoperative PSA was available in 96 patients, with mean $276.2 \mathrm{ng} / \mathrm{mL}$ and median $32.0 \mathrm{ng} / \mathrm{mL}$. Almost $75 \%$ of patients had prostate cancer of Grade Group $\geq 3$ (Gleason Score) $\geq 4+3$.

HGPIN was present together with PCa in 31 (28.4\%) cases. Clinicopathological characteristics of HGPIN cases, shown by ERG-expression status, are summarized in Table 2 .

Of note, only one case showed features of intraductal carcinoma along with HGPIN. The mean age of patients with HGPIN was 74.3 years (range 64-89 years). Preoperative PSA was available in 25 patients, with a median value of $23.2 \mathrm{ng} / \mathrm{mL}$. The frequency of the new PCa Grade Groups in the 31 cases was: Group 1, two(6.5\%); Group 2, seven(22.6\%); Group 3, four $(12.9 \%)$; Group 4, ten $(32.3 \%)$; and Group 5, eight $(25.8 \%)$.

\section{ERG expression}

Associations of ERG immunoreactivity and the clinicopathological characteristics are shown in Table 2. In all cases, endothelial cells showed an appropriate positive internal control, and normal prostatic glands were negative in all cases. Invasive $\mathrm{PCa}$ was positive for ERG in 36 (33\%) patients and negative in $73(67 \%)$ patients. Of the 31 HGPIN cases, 13 (41.9\%) were ERG-positive, while 18 (58.1\%) were ERG-negative. In all 13 cases with ERGpositive HGPIN, the accompanying PCa was ERGpositive as well. Within the ERG-negative HGPIN group (18 cases), accompanying tumors were ERG-negative in $14(77.8 \%)$ cases, and only four $(22.2 \%)$ cases were ERG- 
Table 2 ERG status and clinicopathologic characteristics of Jordanian-Arab prostate cancer patients with HGPIN

\begin{tabular}{|c|c|c|c|}
\hline \multirow[t]{2}{*}{ Variable } & \multicolumn{2}{|l|}{ ERG expression } & \multirow[t]{2}{*}{$P$-value } \\
\hline & Negative $(n=18)$ & Positive $(n=\mid 3)$ & \\
\hline \multicolumn{4}{|l|}{ Age at diagnosis, years } \\
\hline Mean (range) & $74.28(64-89)$ & $76.85(68-90)$ & 0.354 \\
\hline Median (IQR) & $72(68-8 I)$ & $75(7 I-8 I)$ & \\
\hline \multicolumn{4}{|l|}{ Pre-operative PSA, ng/ml } \\
\hline Mean (range) & $248.9(0.1-6758)$ & $78.5(1.1-755.7)$ & 0.889 \\
\hline Median (IQR) & $23.2(8.5-119.1)$ & $18.5(7.0-59.6)$ & \\
\hline \multicolumn{4}{|c|}{ Gleason Score (ISUP 20|4) $(n=3 \mid)$} \\
\hline 3+3 (Grade Group I) & I (50\%) & I (50\%) & 0.517 \\
\hline 3+4 (Grade Group 2) & $2(28.6 \%)$ & $5(71.4 \%)$ & \\
\hline 4+3 (Grade Group 3) & $3(75 \%)$ & I (25\%) & \\
\hline 4+4 (Grade Group 4) & $6(60 \%)$ & $4(40 \%)$ & \\
\hline 4+5/5+5 (Grade Group 5) & $6(75 \%)$ & $2(25 \%)$ & \\
\hline
\end{tabular}

Abbreviation: ISUP, International Society of Urological Pathology.

positive (PCA found in the same core as HGPIN). In the ERG-positive cases, there was no significant heterogeneity for ERG expression in HGPIN and paired PCA. ERG expression was independent of patient age at presentation $(P=0.4)$, preoperative PSA $(P=0.9)$, and $\mathrm{PCa}$ Grade Groups $(P=0.5)$, as shown in Table 2.

\section{Discussion}

The incidence of isolated HGPIN in needle-core biopsies ranges from $<1 \%$ to $24 \% .{ }^{28-32}$ However, reported prevalence of HGPIN in cases with concurrent invasive PCa, evaluated mostly in radical prostatectomy specimens, is much higher $\left(85 \%-100 \% .{ }^{33}\right.$ Most studies to have investigated ERG expression in HGPIN in needle-core biopsies focused on HGPIN as an isolated lesion or in conjunction with PCa. For example, Yaskiv et $\mathrm{al}^{34}$ found HGPIN in 17 of 77 (22\%) cases of limited PCa, and 29\% (five of 17) of HGPIN foci were ERG-positive. van Leenders et $\mathrm{al}^{13}$ reported HGPIN in 21 of 95 (22\%) cases of consecutive patients diagnosed with $\mathrm{PCa}$, of which 52\% (eleven of 21) HGPIN were ERG-positive. In our cohort, HGPIN was found in 31 of 109 (28.4\%) PCa cases, with a rate of ERG positivity of 13 of 31 (41.9\%) foci, which is comparable to these two studies.

Both HGPIN and PCa share several genetic and molecular alterations, eg, similarities include telomere shortening, ${ }^{35}$ chromosomal copy-number alterations involving chromosome 8 and c-myc amplification, ${ }^{36}$ and overexpression of p16. ${ }^{37}$ Probably the most compelling molecular alteration in both HGPIN and $\mathrm{PCa}$ involves TMPRSS2-ERG fusion, the most common genetic alteration found in $\mathrm{PCa}$. Its frequency ranges $50 \%-70 \% .^{7-9}$ TMPRSS2-ERG fusion has also been found in HGPIN, ranging $0-50 \% .{ }^{11-20}$ These differences in incidence of TMPRSS2-ERG fusion in HGPIN might be due to different detection methods (fluorescenceinsitu hybridization, reverse-transcription PCR, IHC) or the type of samples used in these studies (radical prostatectomy versus needle-core biopsy). Much of the data on genetic and molecular similarities between HGPIN and $\mathrm{PCa}$ stems from studies of HGPIN found adjacent to invasive PCa. ${ }^{11-16,19,20,25,38}$ Generally, studies of canceradjacent specimens have shown higher rates of ERG expression: $13 \%-52 \% .{ }^{11-14,16,17}$ Studies performed on isolated HGPIN have found ERG expression that was lower or even absent: $0-5 \% .{ }^{15,38}$

Although the great majority of HGPIN foci in this study were seen adjacent to $\mathrm{PCa}$ in the same tissue core, in a few cases HGPIN foci were seen in cores free of tumors (while cancer was present in the other cores sampled from the same side). HGPIN demonstrated ERG expression in 13 of 31 (41.9\%) patients. In all 13 cases with ERG-positive HGPIN, accompanying PCa was ERGpositive as well. In the 18 ERG-negative cases with HGPIN, accompanying tumors were ERG-negative in 14 (77.8\%) cases, and only four $(22.2 \%)$ cases were ERGpositive. Mosquera et $\mathrm{al}^{20}$ reported similar results: all 22 HGPIN cases with TMPRSS2-ERG gene fusion in their study, and accompanying $\mathrm{PCa}$ shared the same fusion status. On the other hand, of the 120 TMPRSS2-ERG 
fusion-negative HGPIN cases, $32 \%$ (33/102) had TMPRSS2-ERG gene fusion in paired $\mathrm{PCa}$, while the remaining $85 \%$ (102 of 120 ) cases were negative for the fusion gene. Importantly, we found no association between ERG expression in HGPIN and patient age $(P=0.4)$, preoperative PSA $(P=0.9)$, or PCa Grade Group $(P=0.5)$.

The utility of ERG expression in isolated HGPIN has been evaluated in some studies in an attempt to stratify the cancer risk associated with HGPIN. He et al ${ }^{15}$ showed that ERG expression was uncommon in isolated HGPIN (5.3\%) and its expression not associated with increased cancer detection in follow-up biopsies. Gao et $\mathrm{a}^{14}$ suggested that presence of ERG-positive isolated HGPIN in needle-core biopsy can predict subsequent cancer diagnosis, because $95 \%$ of patients with ERG-positive HGPIN were diagnosed with PCa on subsequent biopsies. Park et $\mathrm{al}^{39}$ found that ERG expression was identified in $11.1 \%$ of 461 patients with isolated HGPIN: $53 \%$ of ERG-positive HGPIN in their study were diagnosed with $\mathrm{PCa}$ on follow-up biopsies, in contrast to $35 \%$ of patients with ERG-negative HGPIN. Unfortunately, because our cohort consisted of patients with more advanced disease, reflective of our regional practice environment, we could not evaluate the significance of ERG expression in isolated HGPIN, which is a study limitation.

The high frequency of ERG expression in HGPIN in our study has also been shown in other studies. ${ }^{13,14,19}$ A possible explanation for the high frequency of ERG in these studies and ours is that the HGPIN lesions examined were adjacent to or admixed with PCa. Yaskiv et $\mathrm{al}^{34}$ showed in a cohort of limited PCa in prostate biopsies that ERG was expressed in $29 \%$ of HGPIN immediately adjacent to invasive carcinoma, while it was negative in HGPIN glands away from the PCa. Regarding our patient population in Jordan, there is no national screening program and PSA is not routinely performed, in contrast to PSA-screened, population-based studies from North America and Europe. Most of our patients were older, with $>70 \%$ demonstrating PCa Grade Group 3 or higher, as well as very high PSA values at presentation. Another possibility is that some of the HGPIN lesions could have represented late-stage retrograde spread of adjacent invasive $\mathrm{PCa}$, rather than all of them representing a precursor lesion, as suggested by De Marzo et al. ${ }^{40}$ All of the aforementioned factors possibly influenced the results of ERG expression in our cohort.
Larger studies are certainly needed regarding establishment of the overall frequency of HGPIN and ERG expression in local and regional Arab populations. These studies should include not only cases with PCa but also those with benign, atypical findings, and particularly cases with isolated HGPIN, to estimate more specifically the incidence of HGPIN and prevalence of ERG expression in this population.

\section{Conclusion}

In the current study, we showed that the frequency of HGPIN in Jordanian Arab men with mostly advanced PCa on biopsy was $28.4 \%$. The frequency of ERG expression in HGPIN was $41.9 \%$, which appears similar to some studies conducted in the Western patient population. In the current study, ERG expression was independent of patient age at presentation, preoperative PSA, and PCa Grade Group.

\section{Ethical approval}

This study was approved by the institutional review board (IRB) of King Abdullah University Hospital, Irbid, Jordan. All procedures performed were in accordance with the 1964 Declaration of Helsinki and its later amendments. Because of the retrospective study design, the requirement for patient consent was waived by the IRB. Patient data were kept confidential, and no patient identifiers were included in data files handled for the purposes of this study.

\section{Acknowledgment}

This research was funded by a grant from the Deanship of Research, Jordan University of Science and Technology, Irbid, Jordan.

\section{Disclosure}

The authors report no conflicts of interest in this work.

\section{References}

1. McNeal JE, Bostwick DG. Intraductal dysplasia: a premalignant lesion of the prostate. Human Pathology. 1986;17(1):64-71.

2. Epstein JI. Precursor lesions to prostatic adenocarcinoma. Virchows Archiv. 2009;454(1):1-16. doi:10.1007/s00428-008-0707-5

3. Montironi R, Mazzucchelli R, Lopez-Beltran A, Scarpelli M, Cheng L. Prostatic intraepithelial neoplasia: its morphological and molecular diagnosis and clinical significance. BJU Int. 2011;108(9):1394-1401. doi:10.1111/j.1464-410X.2011.010413.x 
4. Hoedemaeker RF, Kranse R, Rietbergen JB, Kruger AE, Schroder FH, van der Kwast TH. Evaluation of prostate needle biopsies in a population-based screening study: the impact of borderline lesions. Cancer. 1999;85(1):145-152.

5. Abdel-Khalek M, El-Baz M. Ibrahiem el H. Predictors of prostate cancer on extended biopsy in patients with high-grade prostatic intraepithelial neoplasia: a multivariate analysis model. BJU Int. 2004;94(4):528-533. doi:10.1111/j.1464-410X.2004.04996.x

6. Netto GJ, Epstein JI. Widespread high-grade prostatic intraepithelial neoplasia on prostatic needle biopsy: a significant likelihood of subsequently diagnosed adenocarcinoma. Am J Surg Pathol. 2006;30(9):1184-1188. doi:10.1097/01.pas.0000213324.97294.54

7. Magi-Galluzzi C, Tsusuki T, Elson $P$, et al. TMPRSS2-ERG gene fusion prevalence and class are significantly different in prostate cancer of Caucasian, African-American and Japanese patients. Prostate. 2011;71(5):489-497. doi:10.1002/pros.21265

8. Clark J, Merson S, Jhavar S, et al. Diversity of TMPRSS2-ERG fusion transcripts in the human prostate. Oncogene. 2007;26 (18):2667-2673. doi:10.1038/sj.onc.1210070

9. Tomlins SA, Rhodes DR, Perner S, et al. Recurrent fusion of TMPRSS2 and ETS transcription factor genes in prostate cancer. Science (New York, NY). 2005;310(5748):644-648. doi:10.1126/ science. 1117679

10. Aldaoud N, Abdo N, Al Bashir S, et al. Prostate cancer in Jordanian-Arab population: ERG status and relationship with clinicopathologic characteristics. Virchows Archiv. 2017;471:753-759. doi:10.1007/s00428-017-2160-9

11. Cerveira N, Ribeiro FR, Peixoto A, et al. TMPRSS2-ERG gene fusion causing ERG overexpression precedes chromosome copy number changes in prostate carcinomas and paired HGPIN lesions. Neoplasia (New York, NY). 2006;8(10):826-832. doi:10.1593/neo.06427

12. Perner S, Mosquera JM, Demichelis F, et al. TMPRSS2-ERG fusion prostate cancer: an early molecular event associated with invasion. Am J Surg Pathol. 2007;31(6):882-888. doi:10.1097/01. pas.0000213424.38503.aa

13. van Leenders GJ, Boormans JL, Vissers CJ, et al. Antibody EPR3864 is specific for ERG genomic fusions in prostate cancer: implications for pathological practice. Mod Pathol. 2011;24(8):1128-1138. doi:10.1038/modpathol.2011.65

14. Gao X, Li LY, Zhou FJ, et al. ERG rearrangement for predicting subsequent cancer diagnosis in high-grade prostatic intraepithelial neoplasia and lymph node metastasis. Clin Cancer Res. 2012;18 (15):4163-4172. doi:10.1158/1078-0432.CCR-11-2449

15. He H, Osunkoya AO, Carver $\mathrm{P}$, et al. Expression of ERG protein, a prostate cancer specific marker, in high grade prostatic intraepithelial neoplasia (HGPIN): lack of utility to stratify cancer risks associated with HGPIN. BJU Int. 2012;110(11Pt B):E751-E755. doi:10.1111/j.1464-410X.2012.11557.x

16. Tomlins SA, Palanisamy N, Siddiqui J, Chinnaiyan AM, Kunju LP. Antibody-based detection of ERG rearrangements in prostate core biopsies, including diagnostically challenging cases: ERG staining in prostate core biopsies. Arch Pathol Lab Med. 2012;136(8):935-946. doi:10.5858/arpa.2011-0424-OA

17. Lotan TL, Gumuskaya B, Rahimi H, et al. Cytoplasmic PTEN protein loss distinguishes intraductal carcinoma of the prostate from high-grade prostatic intraepithelial neoplasia. Mod Pathol. 2013;26 (4):587-603. doi:10.1038/modpathol.2012.201

18. Verdu M, Trias I, Roman R, et al. ERG expression and prostatic adenocarcinoma. Virchows Archiv. 2013;462(6):639-644. doi:10.1007/s00428-013-1415-3

19. Lee SL, Yu D, Wang C, et al. ERG expression in prostate needle biopsy: potential diagnostic and prognostic implications. Appl Immunohistochem Mol Morphol. 2015;23(7):499-505. doi:10.1097/ PAI.0000000000000119
20. Mosquera JM, Perner S, Genega EM, et al. Characterization of TMPRSS2-ERG fusion high-grade prostatic intraepithelial neoplasia and potential clinical implications. Clin Cancer Res. 2008;14 (11):3380-3385. doi:10.1158/1078-0432.CCR-07-5194

21. Choudhury AD, Eeles R, Freedland SJ, et al. The role of genetic markers in the management of prostate cancer. Eur Urol. 2012;62 (4):577-587. doi:10.1016/j.eururo.2012.05.054

22. Chan SW, Nguyen PN, Violette P, et al. Early detection of clinically significant prostate cancer at diagnosis: a prospective study using a novel panel of TMPRSS2: eTSfusion gene markers. Cancer Med. 2013;2(1):63-75. doi:10.1002/cam4.49

23. Kulda V, Topolcan O, Kucera R, et al. Prognostic significance of TMPRSS2-ERG fusion gene in prostate cancer. Anticancer Res. 2016;36(9):4787-4793. doi:10.21873/anticanres.11037

24. Xu B, Chevarie-Davis M, Chevalier S, et al. The prognostic role of ERG immunopositivity in prostatic acinar adenocarcinoma: a study including 454 cases and review of the literature. Hum Pathol. 2014;45(3):488-497. doi:10.1016/j.humpath.2013.10.012

25. Park K, Tomlins SA, Mudaliar KM, et al. Antibody-based detection of ERG rearrangement-positive prostate cancer. Neoplasia (New York, NY). 2010;12(7):590-598. doi:10.1593/neo.10726

26. Epstein JI, Allsbrook WC Jr., Amin MB, Egevad LL. The 2005 International society of urological pathology (ISUP) consensus conference on gleason grading of prostatic carcinoma. Am J Surg Pathol. 2005;29(9):1228-1242.

27. Epstein JI, Egevad L, Amin MB, Delahunt B, Srigley JR, Humphrey PA. The 2014 International society of urological pathology (ISUP) Consensus conference on gleason grading of prostatic carcinoma: definition of grading patterns and proposal for a new grading system. Am J Surg Pathol. 2016;40(2):244-252. doi:10.1097/PAS.0000000000000530

28. Bostwick DG, Qian J, Frankel K. The incidence of high grade prostatic intraepithelial neoplasia in needle biopsies. $J$ Urol. 1995; 154(5):1791-1794.

29. Vis AN, Van Der Kwast TH. Prostatic intraepithelial neoplasia and putative precursor lesions of prostate cancer: a clinical perspective. BJU Int. 2001;88(2):147-157.

30. Naya Y, Ayala AG, Tamboli P, Babaian RJ. Can the number of cores with high-grade prostate intraepithelial neoplasia predict cancer in men who undergo repeat biopsy? Urology. 2004;63(3):503-508. doi:10.1016/j.urology.2003.09.066

31. Moore CK, Karikehalli S, Nazeer T, Fisher HA, Kaufman RP Jr., Mian BM. Prognostic significance of high grade prostatic intraepithelial neoplasia and atypical small acinar proliferation in the contemporary era. $J$ Urol. 2005;173(1):70-72. doi:10.1097/01. ju.0000148260.69779.c5

32. Antonelli A, Tardanico R, Giovanessi L, et al. Predicting prostate cancer at rebiopsies in patients with high-grade prostatic intraepithelial neoplasia: a study on 546 patients. Prostate Cancer Prostatic Dis. 2011;14(2):173-176. doi:10.1038/pcan.2011.3

33. Ayala AG, Ro JY. Prostatic intraepithelial neoplasia: recent advances. Arch Pathol Lab Med. 2007;131(8):1257-1266.

34. Yaskiv O, Zhang X, Simmerman K, et al. The utility of ERG/P63 double immunohistochemical staining in the diagnosis of limited cancer in prostate needle biopsies. Am J Surg Pathol. 2011;35 (7):1062-1068. doi:10.1097/PAS.0b013e318215cc03

35. Vukovic B, Park PC, Al-Maghrabi J, et al. Evidence of multifocality of telomere erosion in high-grade prostatic intraepithelial neoplasia (HPIN) and concurrent carcinoma. Oncogene. 2003;22 (13):1978-1987. doi:10.1038/sj.onc.1206227

36. Qian J, Jenkins RB, Bostwick DG. Detection of chromosomal anomalies and c-myc gene amplification in the cribriform pattern of prostatic intraepithelial neoplasia and carcinoma by fluorescence in situ hybridization. Mod Pathol. 1997;10(11):1113-1119. 
37. Henshall SM, Quinn DI, Lee CS, et al. Overexpression of the cell cycle inhibitor p16INK4A in high-grade prostatic intraepithelial neoplasia predicts early relapse in prostate cancer patients. Clin Cancer Res. 2001;7(3):544-550.

38. Morais CL, Han JS, Gordetsky J, et al. Utility of PTEN and ERG immunostaining for distinguishing high-grade PIN from intraductal carcinoma of the prostate on needle biopsy. Am J Surg Pathol. 2015;39(2):169-178. doi:10.1097/PAS.0000000000000348
39. Park K, Dalton JT, Narayanan R, et al. TMPRSS2:eRGgene fusion predicts subsequent detection of prostate cancer in patients with high-grade prostatic intraepithelial neoplasia. J Clin Oncol. 2014;32 (3):206-211. doi:10.1200/JCO.2013.49.8386

40. De Marzo AM, Haffner MC, Lotan TL, Yegnasubramanian S, Nelson WG. Premalignancy in prostate cancer: rethin king what we know. Cancer Prevention Research (Philadelphia, Pa). 2016;9(8):648-656. doi:10.1158/1940-6207.CAPR-15-0431

\section{Publish your work in this journal}

Research and Reports in Urology is an international, peer-reviewed, open access journal publishing original research, reports, editorials, reviews and commentaries on all aspects of adult and pediatric urology in the clinic and laboratory including the following topics: Pathology, pathophysiology of urological disease; Investigation and treatment of urological disease; Pharmacology of drugs used for the treatment of urological disease. The manuscript management system is completely online and includes a very quick and fair peer-review system, which is all easy to use. Visit http://www.dovepress.com/ testimonials.php to read real quotes from published authors. 